\title{
Desenvolvimento de kit didático de controle de nível para um sistema tanque simples
}

\author{
Amanda Lisboa Pereira, Luis F. Pozas, Roddy Romero \\ ${ }^{1}$ Curso de Eng. Elétrica - Instituto Federal de Santa Catarina - Câmpus Itajaí \\ Av. Abrahão João Francisco, 3899, Bairro Ressacada, Itajaí/SC - Brazil \\ amanda.lp@aluno.ifsc.edu.br, luis.pozas@ifsc.edu.br, roddy.romerodifsc.edu.br
}

\begin{abstract}
This paper describes a low-cost didactic kit for level control as an auxiliary tool in the study of control systems. With the developed kit, which is composed of hardware elements, the Arduino ${ }^{\odot}$ prototyping platform, and the software Simulink ${ }^{\odot}$, identification tests of first-order systems, obtaining a mathematical model through experimental data, and closed-loop control tests were performed. Through the analysis of the results, it was observed that the identified model presented optimal results in permanent regime, but not very representative in transient regime. As future work, some adjustments to the model were suggested so that the didactic kit can be used in classes.
\end{abstract}

Resumo. O presente trabalho descreve um kit didático de controle de nível de baixo custo, para auxílio ao estudo de sistemas de controle. O kit desenvolvido, o qual é composto por elementos de hardware em conjunto com a plataforma de prototipagem Arduino ${ }^{\odot}$ e associado ao software Simulink ${ }^{\odot}$, permitiu efetuar ensaios de identificação de sistemas de primeira ordem, obtendo-se um modelo matemático através de dados experimentais, e ensaios de controle em malha fechada. Através da análise dos resultados, percebeu-se que o modelo identificado apresentou resultados ótimos em regime permanente, porém pouco representativos em regime transitório. Como trabalhos futuros, foram sugeridos alguns ajustes no modelo para que o kit didático possa ser utilizado nas aulas.

\section{Introdução}

A teoria de sistemas de controle envolve conhecimentos multidisciplinares que abrangem diversos campos das engenharias e da ciência, sendo capaz de lidar com múltiplas variáveis das mais variadas naturezas [Dorf and Bishop 2000]. O controle automático é parte importante de diversos sistemas, como por exemplo sistemas de veículos espaciais, sistemas robóticos, sistemas de manufatura modernos, podendo ser encontrado em quaisquer operações industriais envolvendo controle de variáveis como temperatura, pressão, umidade, fluxo, dentre outras [Ogata 2010].

O monitoramento e controle de nível de fluido em um tanque, é um processo industrial bastante comum, e muito utilizado principalmente em indústrias de processos químicos [Jaafar et al. 2014].

Um sistema tanque simples é um sistema no qual deseja-se controlar a altura de um líquido no interior de um único tanque, através da atuação dinâmica na vazão de entrada (ou alternativamente na vazão de saída, de acordo com a estratégia) [Ponce et al. 2006]. Neste caso específico, a malha de controle é constituída por um dispositivo que atua na 
vazão de entrada (variável manipulada), comandado por um controlador, em resposta a flutuações no líquido do tanque (variável controlada). Por tratar-se de apenas uma única variável monitorada e uma única variável controlada, denomina-se o sistema por SISO (Single Input and Single Output).

O projeto de sistemas de controle SISO dentro da abordagem clássica de controle de processos, traz como requisito conhecer o modelo matemático que descreve o sistema. Dentre algumas possibilidades de obter-se o modelo estão: (a) Modelagem Fenomenológica, a qual apropria-se de análises de balanço de massa e equações diferenciais para descrever o comportamento do sistema, em termos de física [Dorf and Bishop 2000]; (b) a Identificação de Sistemas a partir de dados experimentais, na qual aplica-se uma entrada conhecida ao sistema e observa-se a resposta produzida por tal entrada [Brosilow and Joseph 2002].

Apresenta-se neste trabalho o desenvolvimento de um kit didático acessível, e de baixo custo, para sistemas de controle, que tem por objetivo final auxiliar os docentes no desenvolvimento da respectiva Unidade Curricular. São apresentados resultados obtidos a partir da utilização do kit, nos quais buscou-se identificar o modelo de um sistema tanque simples, a partir de dados experimentais e realizar ensaios com malhas fechadas de controle.

\section{Metodologia}

Apresenta-se nesta seção a metodologia seguida para o desenvolvimento do kit didático. Em um primeiro momento, idealizou-se o uso de recursos para desenvolver um kit de uso efetivo. Entretanto, devido às restrições de acesso ao campus imposta pela situação de pandemia, optou-se por focar o desenvolvimento no conhecimento e adequação dos componentes (bomba, sensores, etc), e na interconexão destes entre si e ao computador. Posteriormente, quando o acesso for regularizado, proceder-se-à ao desenvolvimento da estrutura mecânica de suporte.

O kit provisório é composto de uma estrutura, um reservatório, equipamentos atuadores e sensores, microcontrolador e circuitos auxiliares, conforme apresentado na Figura 1 .

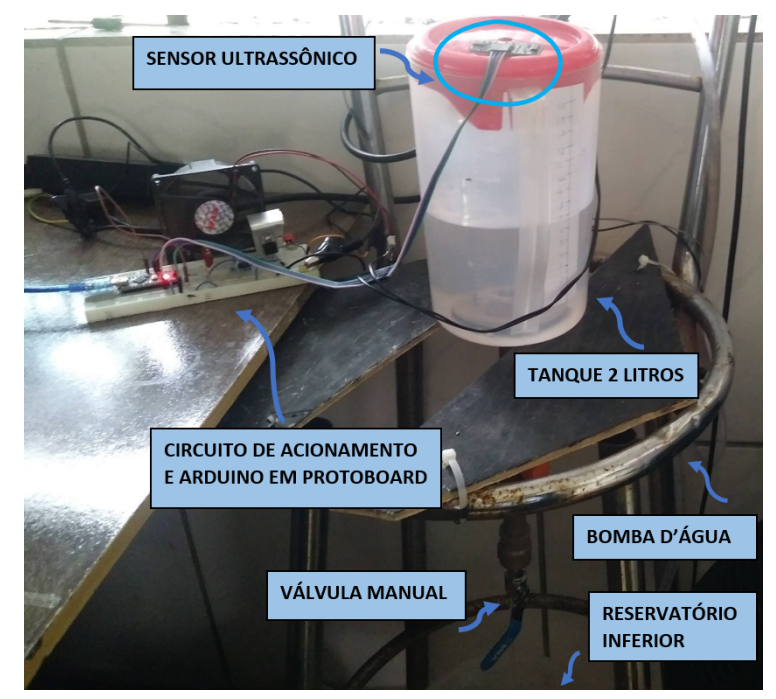

Figura 1. Kit provisório desenvolvido para o estudo de controle de nível 
O reservatório superior (tanque) tem capacidade de aproximadamente 2 litros, com área transversal de $122,72 \mathrm{~cm}^{2}$ e $18 \mathrm{~cm}$ de altura, acrescido de uma válvula manual na saída do tanque (fundo) com a função de ajustar a vazão de saída no tanque, bem como simular perturbações à malha de controle.

Logo abaixo do tanque existe um reservatório de água. Foram acrescentadas duas mangueiras conectadas à bomba: uma mangueira conecta a saída da bomba ao tanque, e a outra conecta a bomba ao reservatório inferior.

\subsection{Hardware e Software utilizados}

Os elementos de Hardware utilizados estão apresentados na Figura 2. Para leitura do nível de água no tanque utilizou-se o sensor ultrassônico HC-SR04, o qual consiste em um sensor digital cujo funcionamento baseia-se na emissão/recepção de um pulso de sinal. O sensor está posicionado na parte superior do tanque.

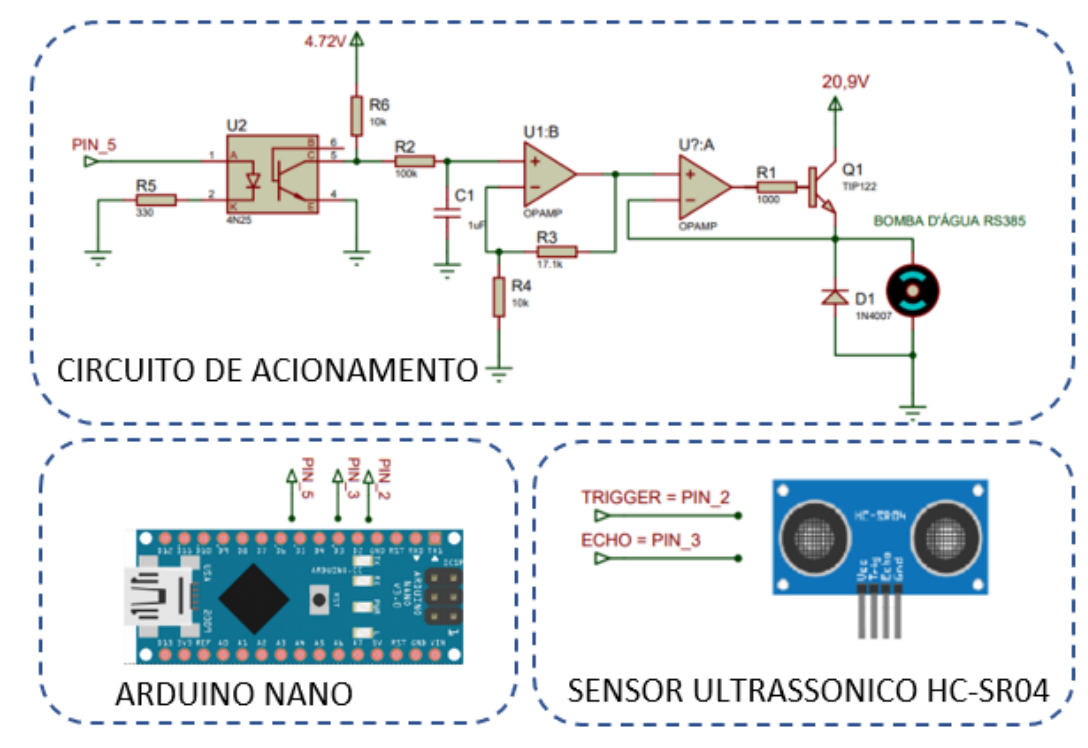

Figura 2. Elementos de hardware utilizados no kit

Já para permitir a atuação junto a vazão de entrada do tanque foi utilizada uma bomba de água comercial $12 \mathrm{Vcc}$, com tensão de operação entre $9 \mathrm{~V}$ e $15 \mathrm{~V}$, e máxima vazão de saída de $2 \mathrm{l} / \mathrm{min}$. Destaca-se que a vazão produzida na saída da bomba responde de maneira linear à variação de tensão de alimentação aplicada em uma boa faixa de operação.

A placa de prototipagem Arduino $\mathrm{Nano}^{\odot}$ foi utilizada para aquisição de dados, atuando em conjunto com o software Simulink ${ }^{\oplus}$ em modo de execução externa em tempo real. A comunicação entre a placa e o software é realizada através do pacote de suporte de hardware para o Arduino ${ }^{\odot}$, o que permite desenvolvimento do sistema através da utilização de diagrama de blocos de uso intuitivo. Desta forma, é feita a interface entre o mundo externo e o computador.

Em relação ao sinal a ser enviado à bomba, após o cálculo de seu valor feito no computador, ele é enviado ao Arduino ${ }^{\odot}$, onde se traduz em um sinal PWM. Este sinal é convertido em um sinal CC médio, com potência adequada através do Circuito de Acionamento. 


\subsection{Validação}

Para validação do kit desenvolvido, foi realizada a identificação de sistemas a partir dos dados experimentais obtidos em ensaio de malha aberta. A partir do modelo matemático foram implementados, através de simulações, controladores proporcional e proporcionalintegral. Posteriormente, novos ensaios foram executados, nos quais cada um dos controladores foram implementados e utilizados no sistema real, visando a comparação das curvas de resposta e erro em malha fechada com as curvas correspondentes obtidas em simulação.

\section{Resultados e Discussão}

Primeiramente efetuou-se o ensaio em malha aberta com o objetivo de realizar a identificação do sistema. O diagrama de blocos desenvolvido para o ensaio é apresenta na Figura 3.
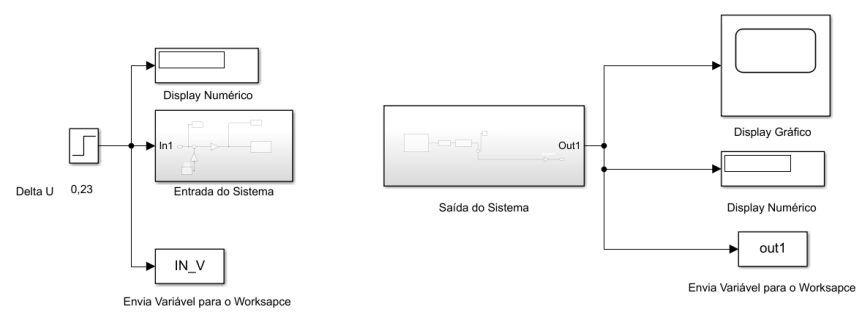

Figura 3. Diagrama de blocos Arduino ${ }^{\odot} /$ Simulink $^{\odot}$

Os blocos de subsistemas presentes no diagrama, denominados entrada e saída, são apresentados na Figura 4.
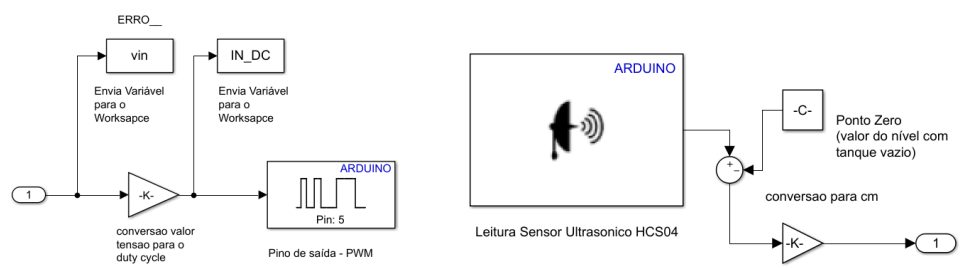

Figura 4. Bloco Entrada a esquerda e bloco Saída a direita

O bloco de entrada do sistema, converte valores de nível de tensão, na faixa de 0 a $12,75 \mathrm{~V}$ para valores na faixa de 0 a 255 , faixa correspondente ao duty-cycle aplicado pelo Arduino $\mathrm{Nano}^{\odot}$. Já o bloco de saída é composto pelo bloco que recebe os valores do sensor ultrassônico, converte-os para centímetros e efetua o ajuste de ponto zero do tanque, o qual representa o valor lido quando o tanque está vazio.

A partir de testes empíricos, nos quais buscou-se ajustar a válvula de saída manual do tanque, foi encontrado o ponto de equilíbrio na altura de $6,35 \mathrm{~cm}$ do tanque, a uma tensão de 9,42V. Após o início do ensaio, foi aplicada uma variação de $0,23 \mathrm{~V}$, em forma de degrau. Ao atingir novo equilíbrio, exibindo comportamento constante no nível do líquido no tanque, verificou-se que a variação de nível obtida foi igual a $3,21 \mathrm{~cm}$. 
O modelo do sistema foi identificado através do método gráfico, no qual primeiramente calculou-se o ganho do sistema $(K)$, através da relação entre a variação da saída e variação da entrada em regime permanente, no valor de 13,96. Definiu-se então a constate de tempo $T$ do sistema, a partir do instante em regime transitório no qual a resposta apresenta 63,2\% de seu valor final, em regime permanente, definindo que $T$ é igual a 319. Como não foi observado atraso de transporte o modelo não contemplou tal parâmetro. $\mathrm{O}$ modelo matemático obtido é representado pela função de transferência no diagrama de blocos no Simulink ${ }^{\odot}$, conforme apresentado na Figura 5.

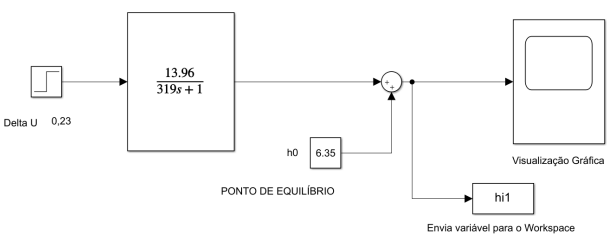

Figura 5. Modelo do sistema em diagrama de blocos

A Figura 6 ilustra a comparação entre a curva obtida a partir do modelo (em vermelho) e a curva de resposta obtida experimentalmente em malha aberta (em azul). Podese afirmar que o modelo comportou-se de maneira satisfatória, apresentando a mesma dinâmica visualizada no teste experimental.

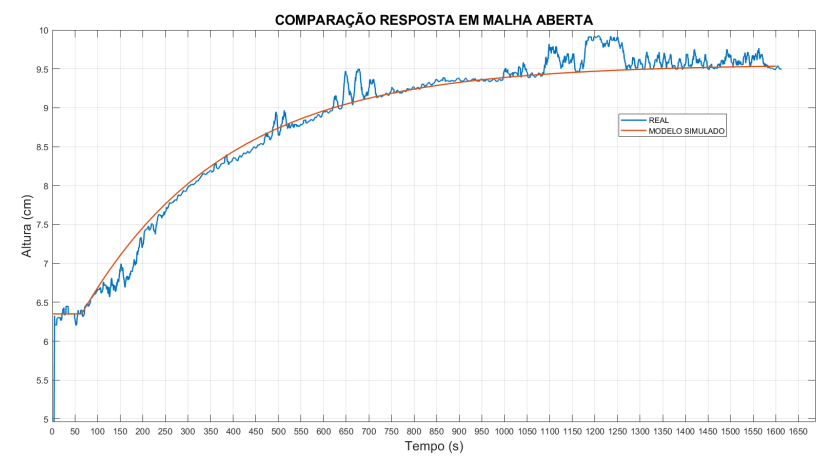

Figura 6. Comparação entre modelo simulado e ensaio em malha aberta

Para o ensaio de malha fechada o digrama de blocos foi modificado conforme é mostrado na Figura 7. O primeiro controlador implementado foi o controlador proporcional com ganho $k_{p}$ igual a 3 . Houve necessidade de alterar o bloco de entrada do sistema, incluindo a informação de tensão de equilíbrio inicial como indicado na Figura 8. Observa-se que o valor de set-point nesse caso foi o valor obtido no ensaio em malha aberta no regime permanente, ou seja o valor de equilíbrio de $6,35 \mathrm{~cm}$ somado à variação de nível obtida de $3,21 \mathrm{~cm}$.

A Figura 9 indica o modelo simulado em malha fechada com set-point definido em 3, 21 uma vez que e o valor de equilíbrio inicial do sistema foi somado posteriormente para visualização no gráfico.

Após o ensaio, as curvas de resposta do sistema e do erro obtido (em azul) foram comparadas com as curvas obtidas em simulação (em vermelho) e são exibidas a seguir 


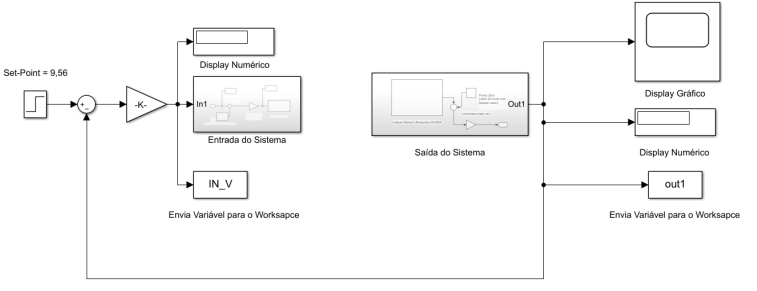

Figura 7. Modificação diagrama de blocos Arduino ${ }^{\odot} /$ Simulink $^{\odot}$

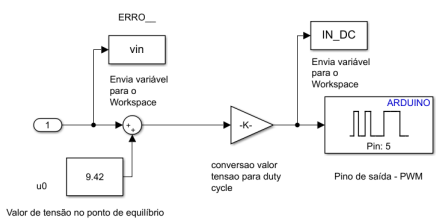

Figura 8. Modificação bloco de entrada Arduino ${ }^{\circledast} /$ Simulink $^{\odot}$

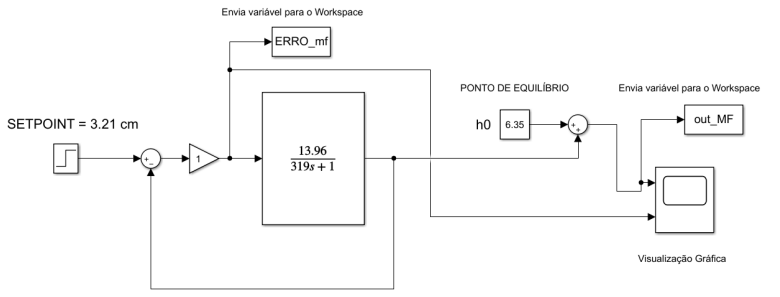

Figura 9. Diagrama de blocos de simulação resposta em malha fechada

nas Figuras 10 e 11. Em regime permanente, ambas as curvas de resposta atingiram valores muito próximos de $9,48 \mathrm{~cm}$, porém a dinâmica apresentada pelas respostas em relação ao regime transitório mostra que, embora as respostas assemelhem-se, o modelo simulado apresenta um tempo de subida menor, e atinge seu valor em regime permanente antes da curva de resposta real do sistema.

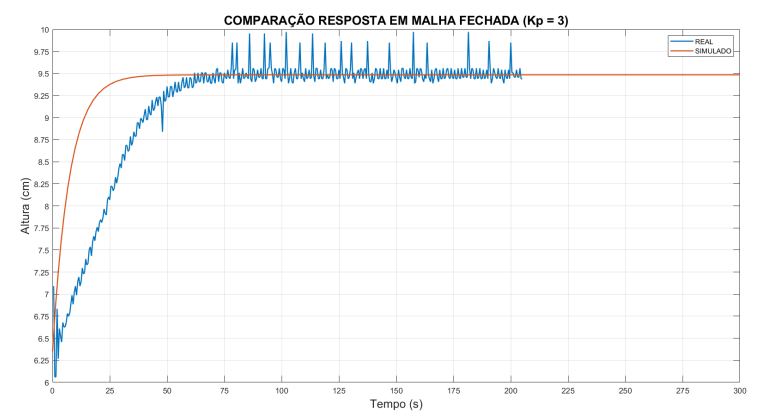

Figura 10. Comparação entre simulação e ensaio de resposta em malha fechada $-\mathrm{Kp}=3$

Por fim, o último ensaio realizado foi através da implementação de controlador proporcional-integral, com as constantes $k p$ no valor de 10 e $t_{i}$ no valor de 1,5. A comparação das respostas é apresentada na Figura 12. Para este caso é necessário observar que o bloco PID utilizado na simulação teve na valor de saída limitado a 12,75, 


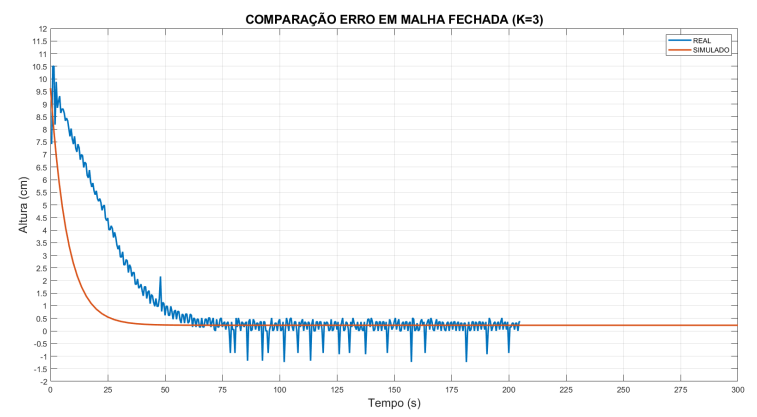

Figura 11. Comparação entre simulação e ensaio de resposta em malha fechada - Erro obtido com $\mathrm{Kp}=3$

uma vez que esse era o maior valor possível de tensão da fonte. Essa limitação aparece na curva do erro obtido, na Figura 13.

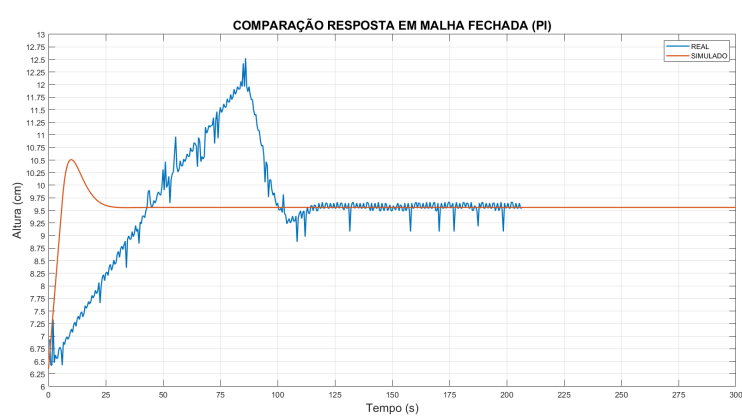

Figura 12. Comparação entre simulação e ensaio - Controlador PI

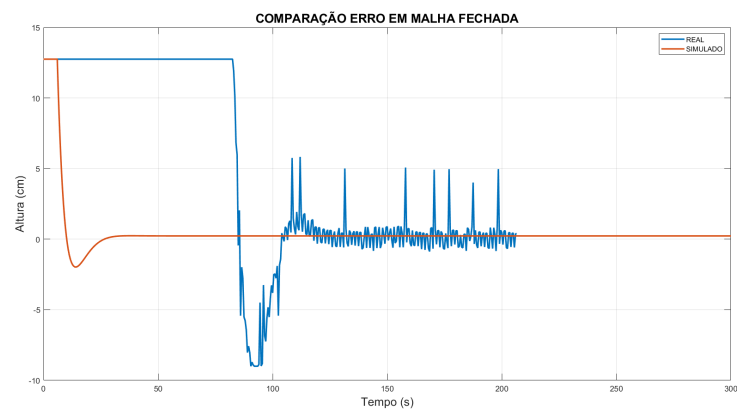

Figura 13. Comparação entre simulação e ensaio - Erro controlador PI

Observa-se neste caso que embora a resposta real tenha apresentado erro tendendo à zero, a partir de 120 segundos, o modelo simulado atingiu tal valor em cerca de 30 segundos. Enquanto na simulação o overshoot ocorreu em cerca de 10 segundos, no valor de 10,5 cm, o sistema real comportou-se de forma distinta, atingindo um overshoot de 12 $\mathrm{cm}$ próximo a 85 segundos.

Percebe-se que em regime permanente as respostas simulada e real apresentam comportamento similar em ambos os casos observados. Contudo, foi possível verificar a inconsistência no regime transitório. Observa-se que a bomba d'água, com a máxima 
tensão de alimentação e consequentemente máxima vazão, não garante a subida do nível tão rápida quanto a subida apresentada em simulação.

Conclui-se que a máxima taxa de variação, o slew-rate, em termos de vazão por centímetros obtida em simulação não condiz com o slew-rate real apresentado pelo sistema. Portanto, é necessário incluir informações no modelo que relacionam os limites físicos da bomba d'água, deixando-o mais complexo porém mais fiel à realidade.

\section{Conclusão}

O kit proposto utiliza a plataforma de prototipagem Arduino ${ }^{\odot}$ atuando em conjunto com o software Simulink ${ }^{\odot}$ tornando possível realizar operações de controle dinâmico atuando em tempo real, controlando a tensão aplicada à bomba d'água para manter o nível de líquido no tanque no valor de set-point desejado.

A partir da identificação do sistema foi possível realizar a implementação de controladores proporcional e proporcional-integral e comparar os resultados simulados com os resultados reais. Constatou-se que o modelo matemático foi muito representativo ao sistema real em relação ao seu comportamento em regime permanente. Contudo, em regime transitório verificou-se que a resposta obtida em simulação distanciou-se da resposta obtida através de ensaios. Portanto, conclui-se que a metodologia utilizada para obtenção do modelo é eficiente em relação ao regime permanente, porém apresentou resultados pouco representativos em regime transitório.

Em trabalhos futuros, será realizada a inclusão de informações respectivas ao comportamento da bomba d'água no desenvolvimento do modelo, mesclando o modelo identificado com a abordagem fenomenológica, visando uma maior representatividade em relação ao sistema real. Outro ponto relevante para trabalhos futuros consiste na inserção de uma resistência para aquecimento da água no interior do tanque, visando adicionar nova malha de controle ao sistema, aumentando as possibilidades e recursos em um único kit didático.

\section{Referências}

Brosilow, C. and Joseph, B. (2002). Techniques of Model-based Control. Prentice-Hall international series in the physical and chemical engineering sciences. Prentice Hall.

Dorf, R. C. and Bishop, R. H. (2000). Modern Control Systems. Prentice-Hall, Inc., USA, 9 th edition.

Jaafar, H. I., Yuslinda, S., Selamat, N., Mohd Aras, M. S., and Rashid, M. (2014). Development of pid controller for controlling desired level of coupled tank system. International Journal of Innovative Technology and Exploring Engineering, 3:32-36.

Ogata, K. (2010). Engenharia de controle moderno. Pearson Prentice Hall, São Paulo, 5 edition.

Ponce, J., Alcantara Ramirez, R., Siller-Alcalá, I., Ramirez-Alpizar, I. G., and NÁJERARAMOS, N. (2006). An educational level control system. 